\title{
Current management options for latent tuberculosis: a review
}

\author{
This article was published in the following Dove Press journal: \\ Infection and Drug Resistance \\ 28 November 2012 \\ Number of times this article has been viewed
}

\section{Brianna L Norton \\ David P Holland}

Department of Medicine, Division of Infectious Diseases, Duke University Medical Center, Durham, NC, USA

Correspondence: David P Holland Box 102359 DUMC, Durham, NC 277I0, USA

$\mathrm{Tel}+19196135160$

Fax + I 9196848902

Email david.p.holland@duke.edu
Abstract: Tuberculosis remains the world's second leading infectious cause of death, with nearly one-third of the global population latently infected. Treatment of latent tuberculosis infection is a mainstay of tuberculosis-control efforts in low-to medium-incidence countries. Isoniazid monotherapy has been the standard of care for decades, but its utility is impaired by poor completion rates. However, new, shorter-course regimens using rifamycins improve completion rates and are cost-saving compared with standard isoniazid monotherapy. We review the currently available therapies for latent tuberculosis infection and their toxicities and include a brief economic comparison of the different regimens.

Keywords: isoniazid, rifampin, rifapentine, tuberculin skin test, interferon-gamma release assay

\section{Introduction}

Tuberculosis (TB) is a global health problem and the second most common infectious cause of death worldwide, ${ }^{1}$ with approximately 8.5-9.2 million incident cases of active tuberculosis in 2010 and 1.2-1.5 million deaths. Furthermore, up to one-third of the world population is chronically infected with Mycobacterium tuberculosis. In this latent tuberculosis infection (LTBI), an individual is infected with the bacteria but does not have symptoms and is unable to spread the bacteria. Instead, the bacilli persist in an inactive state, remaining viable for years or decades. In the vast majority of cases (approximately 90\%), the bacteria remain dormant for the remainder of the individual's life, but about $10 \%$ of infected individuals go on to develop active, contagious tuberculosis. The majority of tuberculosis disease in the United States and other low-prevalence nations is from reactivation of old LTBI rather than recent person-to-person transmission. ${ }^{2,3}$

The goal of LTBI treatment is to eradicate all M. tuberculosis bacilli from the host in order to prevent reactivation, disease, and subsequent transmission. Unfortunately, organisms in latent infection appear to have low metabolic activity, during which antituberculous medications appear to have little efficacy. Therefore, to be effective, treatment of LTBI must be given for extended periods of time.

This requirement for lengthy therapy, as well as the asymptomatic nature of this disease, has a negative impact on treatment completion. For example, in one public health clinic, it was shown that only $26 \%$ of persons identified with LTBI chose to initiate treatment, and only $53 \%$ of that group completed their prescribed regimen. ${ }^{4}$ Clearly, the overall effectiveness of a regimen is severely hindered when completion rates are so low. 
To help improve completion rates, efforts have been made to find more potent drugs with shorter treatment regimens. Isoniazid therapy for 9 months has been the mainstay of treatment since 1965 and has remained the standard of care for LTBI treatment until the last decade. Rifampin for 4 months was added to the United States LTBI guidelines in 2004 as an alternative; however, US guidelines still do not consider it a preferred regimen due to the lack of large efficacy trials. However, the results of a major study published in December $2011^{5}$ may have improved the LTBI efforts by demonstrating the efficacy of a 3-month regimen of weekly rifapentine plus isoniazid. This article will discuss the current management options of LTBI, including the newly added treatment regimen (Table 1).

\section{General principles}

Even after 100 years, diagnosis of LTBI is still most commonly made by a positive tuberculin skin test (TST). ${ }^{6}$ Proper placement is important, following which the patient must return to have the test read within 48-72 hours. When interpreting the test, induration (not erythema) should be measured longitudinally across the forearm, with the measurement recorded in millimeters (including a recording of " $0 \mathrm{~mm}$ " for no induration).

In order to limit treatment to those at highest risk of developing active TB, TST is determined to be "positive" or "negative" based on a patient's risk of exposure in combination with any underlying medical conditions which increase the risk of developing active TB (Table 2). Low-risk patients (for whom testing is not recommended) are considered positive at $15 \mathrm{~mm}$. Patients with moderate risk (eg, hemodialysis-dependent renal failure, diabetes mellitus, recent immigration from an endemic country) are positive at $10 \mathrm{~mm}$. High-risk patients, such as those with human immunodeficiency virus (HIV) infection, are positive at $5 \mathrm{~mm}$. The highest-risk patients (eg, immunosuppressed, contacts to active TB) should be offered treatment regardless of skin-test positivity.

There are several problems with the TST. First, the test is highly operator-dependent, both in placement and in reading; even among highly trained readers, both inter- and intraobserver variability are quite high. ${ }^{7}$

Table I Regimens and doses for treatment of latent tuberculosis infection - guidelines from the Centers for Disease Control and Prevention ${ }^{67,75}$

\begin{tabular}{|c|c|c|c|}
\hline Regimen & Dose & Frequency/duration & Comments \\
\hline \multicolumn{4}{|l|}{ Isoniazid } \\
\hline Adults & $\begin{array}{l}5 \mathrm{mg} / \mathrm{kg} \\
(\max 300 \mathrm{mg})\end{array}$ & Daily $\times 9$ months & Preferred regimen \\
\hline Children & $\begin{array}{l}10-20 \mathrm{mg} / \mathrm{kg} \\
(\max 300 \mathrm{mg})\end{array}$ & & \\
\hline Adults & $\begin{array}{l}15 \mathrm{mg} / \mathrm{kg} \\
(\mathrm{max} 900 \mathrm{mg})\end{array}$ & Twice weekly $\times 9$ months & Directly observed only \\
\hline Children & $\begin{array}{l}20 \mathrm{mg} / \mathrm{kg} \\
(\max 900 \mathrm{mg})\end{array}$ & & \\
\hline \multirow[t]{2}{*}{ Adults } & $\begin{array}{l}5 \mathrm{mg} / \mathrm{kg} \\
(\mathrm{max} 300 \mathrm{mg})\end{array}$ & Daily $\times 6$ months & $\begin{array}{l}\text { Not appropriate for HIV-infected } \\
\text { individuals or children }\end{array}$ \\
\hline & $\begin{array}{l}15 \mathrm{mg} / \mathrm{kg} \\
(\max 900 \mathrm{mg})\end{array}$ & Twice weekly $\times 6$ months & \\
\hline \multicolumn{4}{|l|}{ Rifampin } \\
\hline Adults & $\begin{array}{l}10 \mathrm{mg} / \mathrm{kg} \\
(\max 600 \mathrm{mg})\end{array}$ & Daily $\times 4$ months & \\
\hline Children & $\begin{array}{l}10-20 \mathrm{mg} / \mathrm{kg} \\
(\max 600 \mathrm{mg})\end{array}$ & Daily $\times 6$ months & \\
\hline \multicolumn{4}{|c|}{ Rifapentine/isoniazid } \\
\hline Adults & $\begin{array}{l}\text { Isoniazid } \\
\text { I5 mg/kg } \\
\text { (max } 900 \mathrm{mg} \text { ) plus } \\
\text { Rifapentine } \\
\text { I0.0-14.0 kg: } 300 \mathrm{mg} \\
\text { I4. I-25.0 kg: } 450 \mathrm{mg} \\
25.1-32.0 \mathrm{~kg}: 600 \mathrm{mg} \\
32.1-49.9 \mathrm{~kg}: 750 \mathrm{mg} \\
>50 \mathrm{~kg}: 900 \mathrm{mg}\end{array}$ & Once weekly $\times 3$ months ( 12 doses) & Directly observed only \\
\hline
\end{tabular}

Abbreviation: HIV, human immunodeficiency virus. 
Table 2 Criteria for determining tuberculin skin test positivity ${ }^{76}$

\begin{tabular}{|c|c|c|c|}
\hline Presumptive treatment & $\geq 5 \mathrm{~mm}$ induration & $\geq 10 \mathrm{~mm}$ induration & $\geq 15 \mathrm{~mm}$ induration \\
\hline $\begin{array}{l}\text { HIV-positive or immunosuppressed } \\
\text { patients with recent contact } \\
\text { with active TB case patients } \\
\text { Children under the age } \\
\text { of } 5 \text { years with recent contact } \\
\text { with active TB case patients }{ }^{\mathrm{a}}\end{array}$ & $\begin{array}{l}\text { HIV-positive persons } \\
\text { Recent contact with active } \\
\text { TB case patients } \\
\text { Fibrotic changes on chest } \\
\text { radiograph consistent } \\
\text { with prior TB } \\
\text { Patients with organ transplants } \\
\text { and other immunosuppressed } \\
\text { patients (receiving the } \\
\text { equivalent of } 15 \mathrm{mg} / \mathrm{d} \text { of } \\
\text { prednisone for I month or more) }\end{array}$ & $\begin{array}{l}\text { Persons with the following clinical } \\
\text { conditions: silicosis; diabetes mellitus; } \\
\text { chronic renal failure; leukemia; } \\
\text { lymphoma; carcinoma of the head } \\
\text { or neck; lung cancer; weight loss of } \\
\text { I0\% of ideal body weight; gastrectomy; } \\
\text { and jejunoileal bypass } \\
\text { Children younger than } 4 \text { years of age } \\
\text { Infants, children, and adolescents } \\
\text { exposed to high-risk adults } \\
\text { Recent immigrants (ie, within the } \\
\text { last } 5 \text { years) from high-prevalence } \\
\text { countries } \\
\text { Residents and employees } \\
\text { of the following high-risk congregate } \\
\text { settings: prisons and jails; nursing } \\
\text { homes and other long-term facilities } \\
\text { for the elderly; hospitals and other } \\
\text { health care facilities; residential facilities } \\
\text { for patients with AIDS; and } \\
\text { homeless shelters } \\
\text { Mycobacteriology laboratory personnel }\end{array}$ & $\begin{array}{l}\text { Persons with no } \\
\text { known risk factors } \\
\text { for TB }\end{array}$ \\
\hline
\end{tabular}

Notes: a Children under the age of 5 years who have had contact with an active TB case patient should receive a tuberculin skin test (TST) and a chest radiograph; if there is no evidence of active TB, they should receive window prophylaxis until the result of their second TST is known 8-12 weeks later. If both tests are negative, window prophylaxis can be stopped; otherwise, a full course of LTBI treatment should be given.

Abbreviations: HIV, human immunodeficiency virus; TB, tuberculosis; LTBI, latent tuberculosis infection; AIDS, acquired immunodeficiency syndrome.

Perhaps more importantly, the TST demonstrates a high false-positive rate in patients previously immunized with the Bacille Calmette-Guerin (BCG) vaccine. ${ }^{7}$ US guidelines recommend disregarding $\mathrm{BCG}$ immunization status when interpreting the TST, ${ }^{6}$ as most individuals who have received this vaccine have a high pretest probability of TB exposure.

To counter some of the problems with the TST, a new group of tests, the interferon-gamma release assays (IGRAs), have been developed. There are currently two commercially available tests: the QuantiFERON ${ }^{\circledR}$-TB Gold In-Tube (Cellestis Limited, Chadstone, Australia) and the T-SPOT ${ }^{\circledR}$. TB (Oxford Immunotec, Limited, Abingdon, UK). Both are whole-blood assays that measure interferon-gamma levels after in vitro exposure to TB-specific antigens. They have been shown to predict TB at least as well ${ }^{8}$ or better ${ }^{9,10}$ than the TST, with much greater specificity in patients previously immunized with BCG. ${ }^{11}$ The Centers for Disease Control and Prevention (CDC) has included IGRAs as an option for testing individuals for LTBI in US guidelines. ${ }^{12}$

Before initiating treatment for LTBI, active TB must first be excluded, as single-agent therapy for active disease will quickly select for resistant mutants. Patients should be carefully screened for symptoms of TB (cough for 2 weeks or more, fever, night sweats, weight loss), and a chest radiograph should be performed. In patients from endemic countries, examination of the cervical lymph nodes is not unwarranted, as extrapulmonary TB is more common in these individuals. ${ }^{13}$ Particular attention should be paid to patients with HIV infection, as active TB can be difficult to diagnose in this setting. ${ }^{14}$

If active TB is suspected, the patient should be further evaluated prior to initiation of LTBI treatment. At a minimum, the patient should have three daily sputum specimens cultured for mycobacteria (in addition to site-specific testing for extrapulmonary TB, if indicated).

\section{Isoniazid}

Isoniazid was first introduced as a therapy against M. tuberculosis in the 1950s and was first recommended in US guidelines for LTBI treatment in 1965. It works by inhibition of mycolic acid synthesis, resulting in disruption of the bacterial cell wall. ${ }^{15,16}$ It is rapidly and almost completely absorbed through the gastrointestinal tract, with peak levels 1-2 hours after ingestion, ${ }^{17}$ and distributes to all body tissue, including the cerebrospinal fluid, with concentrations similar to serum. ${ }^{18}$

The first study of isoniazid for treatment of LTBI was the Bethel Alaska Isoniazid study ${ }^{19,20}$ (conducted from 19571959), in which households in 30 communities at high risk 
for tuberculosis were randomly assigned to placebo or daily isoniazid for 12 months. The subjects receiving isoniazid had a $60 \%$ reduction in development of active TB compared with those receiving placebo. Later, a study conducted by the International Union Against Tuberculosis (IUAT) set out to determine what treatment duration was ideal among 27,000 persons with fibrotic pulmonary lesions consistent with old, healed tuberculosis. ${ }^{21}$ This study found a reduction in TB incidence of $21 \%$ in the group taking 3 months of daily isoniazid, $65 \%$ in the group taking 6 months, and $75 \%$ in the group taking 12 months; there was no significant difference in results between the groups undergoing treatment for 6 and 12 months. However, when exclusively analyzing participants that took at least $80 \%$ of the prescribed regimen, the difference in efficacy was substantial, with a $69 \%$ risk reduction in the 6-month arm versus $93 \%$ risk reduction in the 12-month arm.

In order to reconcile these treatment durations, Comstock et $\mathrm{al}^{22}$ reexamined the data from the Bethel Alaska Study, in which the treatment period was ultimately extended for a total of 2 years. A retrospective analysis of case rates among individuals who self-discontinued therapy at varying points in the study showed a significant increase in efficacy among individuals who completed 9 months versus those who only completed 6 months, but those who completed 12 months had a similar case rate to those who only completed 9 months.

As a result, the 9-month duration became the accepted standard of care in the US, and CDC guidelines now recommend isoniazid $5 \mathrm{mg} / \mathrm{kg}$ ( $\max 300 \mathrm{mg}$ ) for 9 months in adults or $10-20 \mathrm{mg} / \mathrm{kg}(\max 300 \mathrm{mg})$ for 9 months in children. ${ }^{6}$ Alternatively, if directly observed therapy is used, adult patients may take $15 \mathrm{mg} / \mathrm{kg}$ twice weekly (max $900 \mathrm{mg}$ ) and children may take $20 \mathrm{mg} / \mathrm{kg}$ (max $900 \mathrm{mg}$ ) twice weekly. The 6-month duration is considered an acceptable alternative for healthy adults with no immunosuppressive conditions (such as HIV infection), see Table 1.

\section{Isoniazid therapy for HIV-positive persons}

Given the syndemics of HIV infection and tuberculosis, especially within the developing world, there has been much interest in demonstrating the efficacy of LTBI treatments among HIV-positive individuals. Many of these studies were conducted in resource-poor nations where the intersection of these epidemics has caused great mortality. Because of increased anergy and lack of T-cell-mediated response to tuberculin skin tests, studies attempted to determine if treatment of latent TB in all HIV-positive patients in areas of endemicity, rather than in TST-positive patients only, could be beneficial. The first meta-analysis was released in 1999 and evaluated seven trials. ${ }^{23}$ The first trial was conducted in Haiti from 1986-1992 and used a regimen of 12 months of isoniazid; this study showed an $83 \%$ reduction among tuberculin positive HIV-infected persons. All other studies in this meta-analysis used 6-month isoniazid therapy versus placebo or no treatment. After pooling data from 4529 subjects, it was found that isoniazid reduced the incidence of active TB by $60 \%$ for persons with a positive TST, but this efficacy was not seen in patients without a positive skin test, who had a nonsignificant reduction in active TB of $16 \%$. A 2010 Cochran review ${ }^{24}$ concluded similar results. In HIV-positive patients with positive tuberculin skin tests, isoniazid therapy reduced the cases of active TB by $62 \%$ as compared with placebo, vs an $11 \%$ reduction for TST-negative subjects. Finally, a recent study in Botswana also showed significant benefit of isoniazid therapy only in patients with a positive TST. ${ }^{25}$

Despite these findings, HIV-positive individuals in lowto medium-incidence countries with direct contact to a case of active tuberculosis are still considered high risk even in the absence of a positive TST. Therefore, the CDC guidelines recommend treating these individuals presumptively; the recommended course of therapy for HIV-positive persons is a 9-month course of isoniazid (with no option for a 6-month course).

\section{Isoniazid toxicity}

The most common toxicity from isoniazid therapy is hepatitis. In the first decade of isoniazid use for active TB and LTBI, this toxicity was not fully recognized. ${ }^{26}$ During this period, there were very few cases of hepatitis reported, most of which were thought to have been caused by concomitant drug administration, such as paraaminosalicylic acid or streptomycin; ${ }^{27,28}$ isoniazid itself was believed to be a rare cause of hepatitis. Subsequently, data from the New York Hospital in 1969 reported that isoniazid alone could cause significant elevations in serum transaminases, ${ }^{29}$ and since that time, multiple studies have shown a true risk of hepatitis and fatality with isoniazid treatment. ${ }^{30,31}$

One of the largest surveillance studies of isoniazid toxicity was conducted in the United States from 1971-1972 among 13,838 patients. $^{32}$ These data showed a risk of isoniazid hepatitis of approximately $1 \%$, associated with increasing age and daily alcohol consumption. Importantly, there were eight deaths attributed to isoniazid hepatitis, all above 35 years old. 
The IUAT study, which evaluated various durations of isoniazid preventative therapy for tuberculosis among 20,840 subjects, found hepatitis in only $0.5 \%$ of subjects, with three cases resulting in death, and an association with increased age. ${ }^{33}$ The US guidelines were then revised to recommend that lowrisk individuals with reactive tuberculin skin tests who were greater than 35 years of age not be treated. The guidelines were further revised in 1983 to recommend baseline and periodic liver function tests for individuals thought to be at higher risk for isoniazid-induced hepatitis, including those greater than 35 years of age and HIV-positive patients.

Since this time, multiple studies have shown that the risk of hepatitis may not be as great as previously reported, particularly with close patient monitoring. One large review that included 202,497 patients, found only two deaths $(0.001 \%)$ among patients selected and monitored as per the 1983 guidelines. ${ }^{34}$ Since, some studies have shown that clinical monitoring alone is sufficient to reduce the risk of isoniazid-induced hepatitis. A 7-year survey of 11,141 patients receiving isoniazid therapy for latent tuberculosis who were only monitored clinically found a hepatitis rate of only $0.15 \%$, associated with an increase in age, and there were no cases of death. ${ }^{35}$ Of note, only $20 \%$ of patients included in the study were greater than 35 years old. Other studies have shown that many health departments in the United States have solely used clinical monitoring as a means for early detection of isoniazid-induced hepatitis, with good success and continued low rates of hepatitis and fatal disease. ${ }^{36}$

The risk of hepatitis does not appear to be much increased in the HIV-positive population, though many studies have been conducted with patients who were not taking concomitant antiretroviral therapy. The most current guidelines by the American Thoracic Society and CDC recommend baseline transaminase testing in only a select group of patients believed to be at higher risk for hepatitis, such as patients with HIV disease, history of liver disease, or alcoholism; there are no specific recommendations concerning age. However, all patients should be monitored clinically at least once per month for signs and symptoms of hepatitis.

Another potential side effect of isoniazid is peripheral neuropathy, which occurs in $2 \%$ of patients due to its interference in the metabolism of pyridoxine. ${ }^{37,38}$ There is limited data on its frequency and prevention, but it is recommended that patients at greater susceptibility to peripheral neuropathy, such as patients with alcoholism, HIV disease, diabetes mellitus, or who are pregnant, take pyridoxine supplements when receiving isoniazid therapy.

\section{Rifampin}

Rifampin inhibits bacterial RNA synthesis by binding to the beta subunit of DNA-dependent RNA polymerase, blocking RNA transcription. It is fairly well absorbed with peak serum concentrations at 2-4 hours and is primarily metabolized through the liver with a half-life elimination of 3-4 hours. ${ }^{17}$ It has been considered in the treatment of LTBI because of its potent bactericidal activity against $M$. tuberculosis. In an early mouse model of latent TB, a 3-month course of rifampin was significantly better than 6 months of isoniazid at sterilizing mouse spleen. ${ }^{39}$

The only randomized clinical trial evaluating the efficacy of rifampin in preventing tuberculosis reactivation was conducted in Hong Kong in a group of patients with silicosis residing in an area with high rates of tuberculosis. ${ }^{40}$ The subjects were randomized to 6 months of isoniazid, 3 months of rifampin, 3 months of isoniazid plus rifampin, or placebo. All regimens were statistically and clinically superior to placebo, though no treatment group performed better than another. Adverse events were similar in all groups, with only one person (who received isoniazid monotherapy) acquiring symptomatic hepatitis. Based on these results, 3 months of rifampin was considered equivalent to 6 months of isoniazid; because 9 months of isoniazid is the standard of care, the recommended duration for rifampin is 4 months (extended to 6 months in children), see Table $1 .^{6}$

Additional evidence on rifampin efficacy was provided by a retrospective cohort study conducted in Boston in 1984 among 204 homeless individuals exposed to isoniazid-resistant tuberculosis. ${ }^{41}$ Recent skin converters were assigned to isoniazid, rifampin, or isoniazid plus rifampin based on provider preference; some subjects refused therapy. Six of $71(8.6 \%)$ individuals who received no therapy, three of 38 (7.9\%) in the isoniazid monotherapy group, and none in the rifampin or rifampin plus isoniazid groups (49 and 37 persons, respectively) developed active tuberculosis.

Despite the paucity of data regarding rifampin, the lack of randomized, controlled trials of rifampin efficacy may not be terribly important. In contrast to the lack of efficacy data, there have been multiple studies demonstrating that rifampin has markedly better completion rates than isoniazid. ${ }^{42-44}$ Because adherence strongly influences the overall effectiveness of a regimen, it has been shown that even if rifampin were less efficacious than isoniazid (by up to $17 \%$ ), it is still more effective and less expensive 
than isoniazid. ${ }^{45}$ Therefore, at least on the public health scale, rifampin may offer significant advantages to isoniazid in the treatment of LTBI.

It is particularly important to exclude active tuberculosis before initiating LTBI treatment with rifampin, as rifampinresistant TB requires lengthy and expensive treatment. ${ }^{46}$ Unlike isoniazid, in which no study has demonstrated acquired resistance during LTBI treatment (when active TB had been excluded beforehand), a single report has documented a patient on rifampin monotherapy developing active TB with rifampin resistance. ${ }^{47}$

\section{Rifampin therapy for HIV-positive persons}

Although there are no trials that specifically evaluate rifampin monotherapy in an HIV-infected population, US guidelines list rifampin as an acceptable alternative to isoniazid monotherapy in this population.

In a study of 1148 HIV-positive persons in South Africa, patients with a positive tuberculin test were randomized to rifampin plus isoniazid twice weekly for 3 months, weekly rifapentine plus isoniazid for 3 months, isoniazid monotherapy daily continuously for the duration of the study, or isoniazid daily for 6 months; combination therapies were given by directly observed therapy (DOT). ${ }^{48}$ All regimens conferred a similar protection against reactivation of tuberculosis, but more hepatotoxicity was seen in the continuous isoniazid arm. It is important to note that none of these patients were taking antiretroviral therapy.

Finally, a large Cochrane meta-analysis ${ }^{24}$ published in 2010 was performed on 12 controlled trials among 8578 subjects who received regimens that included isoniazid, isoniazid plus rifampin, rifampin plus pyrazinamide, and isoniazid/rifampin/pyrazinamide. All regimens reduced the risk of reactivation of tuberculosis as compared with placebo. There was a trend towards greater adverse events among the combination arms.

These trials have demonstrated that rifampin appears to be well tolerated and efficacious among HIV-positive persons, at least in combination with isoniazid. However, rifampin plus pyrazinamide has been shown to have unacceptably high rates of severe hepatotoxicity and is no longer recommended (see below). ${ }^{49}$

The primary limitation to using rifampin monotherapy in patients with HIV infection is interactions with many commonly used antiretrovirals, particularly protease inhibitors. ${ }^{50}$ If such drug interactions preclude the use of rifampin, rifabutin (which has less potent effects on the cytochrome P-450 system) can be substituted. ${ }^{6}$

\section{Rifampin toxicity}

Many randomized control trials have evaluated the safety of rifampin for LTBI, and all have shown rifampin to be as safe or safer ${ }^{36,43,51}$ than isoniazid. A meta-analysis comparing 4 months of rifampin with 9 months of isoniazid found that rates of hepatotoxicity were significantly less in the rifampin group $(0 \%-0.7 \%)$ compared with the isoniazid group $(1.4 \%-5.2 \%) .^{52}$

Rifampin can also cause a hypersensitivity syndrome characterized by flu-like symptoms and/or thrombocytopenia; interstitial nephritis can also occur with rifampin. ${ }^{53-55}$ These symptoms are more common with intermittent dosing than with daily dosing but should be considered in any patient on rifampin who presents with fever, purpura, or bleeding.

As noted above, rifampin has profound effects on the cytochrome P-450 system, so a thorough check of drug interactions is always warranted when patients are started on rifampin. In addition to antiretrovirals, common drugs with significant interactions include warfarin, oral contraceptives, and digoxin.

\section{Rifampin plus pyrazinamide}

Although the exact mechanism of action of pyrazinamide is unknown, it is clear that the drug has activity against dormant/ semidormant $M$. tuberculosis bacilli. ${ }^{56}$ This feature gives it potent sterilizing activity and, along with rifampin, provides for the current "short-course" therapy for active TB. ${ }^{46}$

All efficacy trials for the prevention of active tuberculosis with rifampin plus pyrazinamide have been conducted in HIVpositive patients. In the first study of this regimen, a 2-month course of rifampin plus pyrazinamide proved comparable to 12 months of isoniazid in preventing active $\mathrm{TB}$, and side effects were minimal. ${ }^{57}$ Guidelines were changed to include a recommendation of 2 months of rifampin plus pyrazinamide as an alternative to the standard isoniazid regimen.

Unfortunately, unforeseen cases of fatal hepatotoxicity began to arise, ${ }^{58}$ and two deaths were reported. ${ }^{59} \mathrm{~A}$ second randomized control trial of $589 \mathrm{HIV}$-negative subjects compared toxicities between persons taking 2 months of rifampin plus pyrazinamide versus 6 months of isoniazid. ${ }^{60}$ There was more grade 3 and 4 hepatotoxicity in the combination therapy group as compared with the group taking isoniazid $(7.7 \%$ versus $1 \%)$, and more patients discontinued rifampin/pyrazinamide due to adverse events.

In light of these reports, the CDC conducted a surveillance study of persons taking rifampin plus pyrazinamide for LTBI treatment from October 2000 to June $2003 .{ }^{49}$ There were 48 cases of reported severe 
hepatotoxicity (hospitalization or death), of which eleven were fatal. Of 7737 patients who were reported to have started rifampin plus pyrazinamide for treatment of LTBI during the survey period, 146 patients discontinued the regimen because of symptoms of hepatitis (rate: 18.9 per 1000 treatment initiations; 95\% CI: 17.4-20.4), a toxicity rate much greater than seen with isoniazid treatment. In August 2003, the CDC guidelines were revised to state that rifampin/pyrazinamide was not a recommended treatment option for LTBI therapy due to unacceptably high rates of hepatotoxicity and death.

\section{Rifapentine plus isoniazid}

Rifapentine is a long-acting rifamycin that has been studied for use in tuberculosis treatment. Rifapentine has the potency of rifampin, with a low minimum inhibitory concentration to M. tuberculosis, but a half-life five times longer. ${ }^{61}$ In a mouse model of LTBI, when compared to other rifamycins (rifampin and rifabutin), rifapentine had both the highest peak serum levels and the longest half-life (23.4 hours); 6 days after delivery of the drug, the serum concentrations of rifapentine were still above the minimum inhibitory concentration. ${ }^{62}$ Furthermore, the bactericidal activity of rifapentine in mice after 6 weeks of treatment was the same as that after 12 weeks of rifampin treatment at the same dose and rate. The robust potency and long half-life obviously make this drug alluring as a treatment for LTBI.

In another murine model of LTBI, weekly rifapentine versus weekly rifapentine plus isoniazid were compared to daily isoniazid. ${ }^{63}$ The results of this study showed that 18 doses of once-weekly rifapentine plus isoniazid were comparable to 18 weeks of daily isoniazid in terms of organ sterilization and organism-load reduction. Monotherapy with weekly rifapentine, however, did not offer advantages even over placebo. Other LTBI murine models confirmed these findings. ${ }^{64,65}$

In 2006, the first human clinical trial using rifapentine was published. ${ }^{66}$ Household contacts of active tuberculosis were given weekly rifapentine plus isoniazid or daily rifampin plus pyrazinamide. Unfortunately, the study was halted early due to unacceptably high toxicity in the control regimen (rifampin/pyrazinamide). Though the study was not powered as intended, very few cases of active TB developed in either arm of the study, and there were no difference in outcome between either regimen. Side effects and tolerability were better in the isoniazid/rifapentine arm of the study. A second study from South Africa compared rifapentine plus isoniazid with four other regimens in patients with HIV infection and found it to be equally effective to standard isoniazid monotherapy. ${ }^{48}$

Most recently, the $\mathrm{CDC}$ published the results of a large randomized, controlled trial (PREVENT-TB) ${ }^{5}$ with 7731 participants, providing further evidence that rifapentine plus isoniazid given weekly for 3 months was just as effective as the standard daily isoniazid for 9 months in preventing active tuberculosis in high-risk individuals. The subjects in the combination arm received directly observed therapy, while the subjects in the isoniazid monotherapy arm selfadministered their medication. All subjects were considered high risk, including close contacts to persons with active tuberculosis, recent skin test converters, and persons with HIV infection; subjects were followed at least 2 years after completing therapy. The cumulative proportion of subjects that developed active TB was $0.19 \%$ (7/3986) in the isoniazid/rifapentine arm compared with $0.43 \%$ (15/3745) in the isoniazid monotherapy arm, meeting criteria for noninferiority. More subjects in the 3-month combination regimen completed their therapy versus 9 months of isoniazid ( $82 \%$ vs $69 \%, P<0.001)$. The percentage of patients with any adverse event was lower in the combination-therapy group, and more patients acquired treatment-related hepatotoxicity in the isoniazid monotherapy group than the rifapentine/isoniazid group $(2.7 \%$ vs $0.4 \%, P<0.001)$.

In response to this study, the $\mathrm{CDC}$ has now updated the US guidelines for LTBI treatment to include a once-weekly, rifapentine-plus-isoniazid regimen given for 3 months (12 doses) in adults and children over 12 years old, though it can be used in children as young as 2 years of age on a case-by-case basis. ${ }^{67}$ However, the guidelines also specify that this regimen should be given only with DOT. While a shorter, less toxic regimen for LTBI has the potential to considerably affect the active tuberculosis cases in countries with low to moderate $\mathrm{TB}$ incidence, the requirement for DOT will be a large financial hurdle to overcome for most programs; however, for the highest-risk patients this cost may be justified. ${ }^{68}$ Currently there is a trial being conducted that evaluates the adherence and cost effectiveness of selfadministered weekly rifapentine plus isoniazid.

\section{Rifapentine plus isoniazid in HIV-infected persons}

The PREVENT-TB study only included $2.6 \%$ HIV positive persons, approximately $100 \mathrm{HIV}$-infected subjects in each arm of the trial, so data from this study on the safety and efficacy of rifapentine plus isoniazid in HIV-positive patients is limited. 
However, all subjects in the South African study were HIV-positive. ${ }^{48}$ In that study, the efficacy of rifapentine plus isoniazid was similar to isoniazid monotherapy with fewer side effects, but of the three cases of multiple-drug resistant TB (MDR-TB) (defined as resistance to both isoniazid and rifampin) that developed during the study, two occurred in the isoniazid/rifapentine group. That said, the overall incidence of MDR-TB in that study was similar to the background rate of MDR-TB in South Africa, suggesting that the therapy did not select for resistant mutants. However, when using this regimen in patients with HIV infection, it is particularly important to exclude active TB before initiating therapy.

Like rifampin, rifapentine is a potent inhibitor of the cytochrome P-450 system and therefore interacts with a number of antiretroviral agents. All studies of rifapentine in HIV-positive patients conducted to date have excluded patients on antiretroviral therapy, so the CDC guidelines for the use of rifapentine plus isoniazid include only HIV patients not taking antiretrovirals.

\section{Rifapentine plus isoniazid toxicity}

In the PREVENT-TB study, isoniazid monotherapy was associated with significantly greater hepatotoxicity than the combination treatment ( $2.7 \%$ versus $0.4 \%)$. However, rifapentine plus isoniazid was associated with significantly greater hypersensitivity reactions ( $3.8 \%$ vs $0.5 \%)$. Of note, the hypersensitivity syndrome associated with rifamycins is not well-defined, so reporting in the study was somewhat subjective. The incidence of this syndrome declined substantially over the course of this trial, and in other studies hypersensitivity to rifapentine was not reported, suggesting that there may have been a reporting bias. Nonetheless, rifamycin hypersensitivity can be serious and should be considered in any patient on rifapentine presenting with a compatible syndrome.

As with all rifamycins, drug interactions are a considerable problem due to the induction of the cytochrome P450 enzyme system, so careful attention should be paid to any concomitant medications.

\section{Treatment of latent multiple-drug resistant tuberculosis}

Contacts to cases of MDR-TB warrant special attention as the approach to these individuals is not clear. No regimen for treatment of latent MDR-TB infection has been studied in a randomized, controlled trial. CDC guidelines recommend pyrazinamide in combination with either ethambutol or a fluoroquinolone, ${ }^{6}$ but the utility of these regimens has been limited by high rates of intolerability and toxicity. ${ }^{69-72}$ A fluoroquinolone alone or in combination with ethambutol may be a better option, ${ }^{45}$ but clearly, any regimen should take into consideration the susceptibility profile of the source case.

Another option for otherwise low-risk individuals (ie, those with no medical risk for activation, such as HIV infection) is close observation. This recommendation is based on the fact that the vast majority of individuals infected with TB will never develop active $\mathrm{TB}^{73}$ and on the current lack of any definitive treatment.

\section{Conclusion}

Treatment of LTBI is a mainstay of TB control efforts in the $\mathrm{US}^{6}$ and other developed economies. While treatment is generally safe and effective, its overall utility is limited by the requirement for long courses of treatment in patients who are otherwise well. Fortunately, new shorter-course regimens have the potential to significantly improve completion rates. Isoniazid plus rifapentine holds promise, though the expense of DOT may be a major hurdle for some programs. Meanwhile, 4 months of rifampin has been shown to be cost-saving (less expensive and more effective) compared with both isoniazid and isoniazid/rifapentine and should be considered in individuals where drug interactions are not a concern. Studies of even shorter (1 month) regimens are underway, as well as studies of self-administered rifapentine plus isoniazid, either of which would further improve TB control efforts.

One area that could have a major impact on LTBI treatment strategies would be an improvement in diagnostics. Neither the TST nor the interferon-gamma release assays are good predictors of TB activation, which is the primary outcome of interest. A test that would enable providers to determine which patients are most likely to develop active TB would allow programs to focus their efforts on only the highest-risk individuals, preventing wasteful treatment of low-risk individuals and avoiding unnecessary exposure of healthy patients to potentially toxic drugs.

As TB incidence continues to decline in the US, ${ }^{74}$ it will be increasingly difficult to identify patients at the most risk for active TB. This effort has historically been led by public health programs, but in the future, interventions by private health care providers will be essential in testing patients for LTBI and providing appropriate therapy. Hopefully, new diagnostic modalities and shorter-course therapies will support this transition, ultimately leading to TB eradication. 


\section{Disclosure}

The authors report no conflicts of interest in this work.

\section{References}

1. World Health Organization. World Health Statistics 2012. Geneva: World Health Organization; 2012. Available from: http://www.who.int/ gho/publications/world_health_statistics/2012/en/index.html. Accessed June 13, 2012.

2. Weis SE, Pogoda JM, Yang Z, et al. Transmission dynamics of tuberculosis in Tarrant county, Texas. Am J Respir Crit Care Med. 2002; 166(1):36-42.

3. Small PM, Hopewell PC, Singh SP, et al. The epidemiology of tuberculosis in San Francisco. A population-based study using conventional and molecular methods. N Eng J Med. 1994;330(24): 1703-1709.

4. Goswami ND, Gadkowski LB, Piedrahita C, et al. Predictors of latent tuberculosis treatment initiation and completion at a US public health clinic: a prospective cohort study. BMC Public Health 2012;12(1):468.

5. Sterling TR, Villarino ME, Borisov AS, et al; TB Trials Consortium PREVENT TB Study Team. Three months of rifapentine and isoniazid for latent tuberculosis infection. $N$ Eng $J$ Med. 2011;365(23): 2155-2166.

6. Centers for Disease Control and Prevention (CDC). Targeted tuberculin testing and treatment of latent tuberculosis infection. MMWR Recomm Rep. 2000;49(RR-6):1-51.

7. Pouchot J, Grasland A, Collet C, Coste J, Esdaile JM, Vinceneux P. Reliability of tuberculin skin test measurement. Ann Intern Med. 1997; 126(3):210-214.

8. Bakir M, Millington KA, Soysal A, et al. Prognostic value of a T-cell-based, interferon-gamma biomarker in children with tuberculosis contact. Ann Intern Med. 2008;149(11):777-787.

9. Diel R, Loddenkemper R, Meywald-Walter K, Niemann S, Nienhaus A. Predictive value of a whole blood IFN-gamma assay for the development of active tuberculosis disease after recent infection with Mycobacterium tuberculosis. Am J Respir Crit Care Med. 2008;177(10): 1164-1170.

10. Leung CC, Yam WC, Yew WW, et al. T-Spot.TB outperforms tuberculin skin test in predicting tuberculosis disease. Am J Respir Crit. Care Med. 2010;182(6):834-840.

11. Pai M, Zwerling A, Menzies D. Systematic review: T-cell-based assays for the diagnosis of latent tuberculosis infection: an update. Ann Intern Med. 2008;149(3):177-184.

12. Mazurek GH, Jereb J, Vernon A, et al; Centers for Disease Control and Prevention (CDC). Updated guidelines for using Interferon Gamma Release Assays to detect Mycobacterium tuberculosis infection - United States, 2010. MMWR Recomm Rep. 2010;59(RR-5):1-25.

13. Kipp AM, Stout JE, Hamilton CD, Van Rie A. Extrapulmonary tuberculosis, human immunodeficiency virus, and foreign birth in North Carolina, 1993-2006. BMC Public Health. 2008;8:107.

14. Centers for Disease Control and Prevention (CDC). Prevention and treatment of tuberculosis among patients infected with human immunodeficiency virus: principles of therapy and revised recommendations. MMWR Recomm Rep. 1998;47(RR-20): $1-58$.

15. Takayama K, Wang L, David HL. Effect of isoniazid on the in vivo mycolic acid synthesis, cell growth, and viability of mycobacterium tuberculosis. Antimicrob Agents and Chemother. 1972;2(1):29-35.

16. Winder FG, Collins PB. Inhibition by isoniazid of synthesis of mycolic acids in mycobacterium tuberculosis. J Gen Microbiol. 1970;63(1): $41-48$.

17. Holdiness MR. Clinical pharmacokinetics of the antituberculosis drugs. Clin Pharmacokinet. 1984;9(6):511-544.

18. Weber WW, Hein DW. Clinical pharmacokinetics of isoniazid. Clin Pharmacokinet. 1979;4(6):401-422.
19. Comstock GW, Baum C, Snider DE Jr. Isoniazid prophylaxis among Alaskan Eskimos: a final report of the bethel isoniazid studies. Am Rev Respir Dis. 1979;119(5):827-830.

20. Comstock GW, Ferebee SH, Hammes LM. A controlled trial of community-wide isoniazid prophylaxis in Alaska. Am Rev Respir Dis. 1967;95(6):935-943.

21. Efficacy of various durations of isoniazid preventive therapy for tuberculosis: five years of follow-up in the IUAT trial. International Union Against Tuberculosis Committee on Prophylaxis. Bull World Health Organ. 1982;60(4):555-564.

22. Comstock GW. How much isoniazid is needed for prevention of tuberculosis among immunocompetent adults? Int J Tuberc Lung Dis. 1999;3(10):847-850.

23. Pape JW, Jean SS, Ho JL, Hafner A, Johnson WD Jr. Effect of isoniazid prophylaxis on incidence of active tuberculosis and progression of HIV infection. Lancet. 1993;342(8866):268-272.

24. Akolo C, Adetifa I, Shepperd S, Volmink J. Treatment of latent tuberculosis infection in HIV infected persons. Cochrane Database Syst Rev. 2010;1:CD000171.

25. Samandari T, Agizew TB, Nyirenda S, et al. 6-month versus 36-month isoniazid preventive treatment for tuberculosis in adults with HIV infection in Botswana: a randomised, double-blind, placebo-controlled trial. Lancet. 2011;377(9777):1588-1598.

26. Ferebee SH, Mount FW. Tuberculosis morbidity in a controlled trial of the prophylactic use of isoniazid among household contacts. Am Review Respir Dis. 1962;85:490-510.

27. Ockerman PA, Ryde C. Serum transaminase levels in pulmonary tuberculosis. Effect of triple treatment with 4-4 1-diisoamyloxythiocarbanilide or PAS in combination with streptomycin and isoniazid. Scand J Respir Dis. 1968;49(1):35-41.

28. Berte SJ, Dimase JD, Christianson CS. Isoniazid, para-aminosalicylic acid, and streptomycin intolerance in 1,744 patients. An analysis of reactions to single drugs and drug groups plus data on multiple reactions, type and time of reactions, and desensitization. Am Rev Respir Dis. 1964; 90:598-606.

29. Scharer L, Smith JP. Serum transaminase elevations and other hepatic abnormalities in patients receiving isoniazid. Ann Intern Med. 1969;71(6):1113-1120.

30. Mitchell JR, Zimmerman HJ, Ishak KG, et al. Isoniazid liver injury: clinical spectrum, pathology, and probable pathogenesis. Ann Intern Med. 1976;84(2):181-192.

31. Byrd RB, Horn BR, Solomon DA, Griggs GA. Toxic effects of isoniazid in tuberculosis chemoprophylaxis. Role of biochemical monitoring in 1,000 patients. JAMA. 1979;241(12):1239-1241.

32. Kopanoff DE, Snider DE Jr, Caras GJ. Isoniazid-related hepatitis: a US Public Health Service cooperative surveillance study. Am Rev Respir Dis. 1978;117(6):991-1001.

33. Efficacy of various durations of isoniazid preventive therapy for tuberculosis: five years of follow-up in the IUAT trial. International Union Against Tuberculosis Committee on Prophylaxis. Bull World Health Organ. 1982;60(4):555-564.

34. Salpeter SR. Fatal isoniazid-induced hepatitis. Its risk during chemoprophylaxis. West J Med. 1993;159(5):560-564.

35. Nolan CM, Goldberg SV, Buskin SE. Hepatotoxicity associated with isoniazid preventive therapy: a 7-year survey from a public health tuberculosis clinic. JAMA. 1999;281(11):1014-1018.

36. LoBue PA, Moser KS. Use of isoniazid for latent tuberculosis infection in a public health clinic. Am J Respir Crit Care Med. 2003;168(4): 443-447.

37. Oestreicher R, Dressler SH, Middlebrook G. Peripheral neuritis in tuberculous patients treated with isoniazid. Am Rev Tuberc. 1954; 70(3):504-508.

38. Snider DE Jr. Pyridoxine supplementation during isoniazid therapy. Tubercle. 1980;61(4):191-196.

39. Lecoeur HF, Truffot-Pernot C, Grosset JH. Experimental short-course preventive therapy of tuberculosis with rifampin and pyrazinamide. Am Rev Respir Dis. 1989;140(5):1189-1193. 
40. A double-blind placebo-controlled clinical trial of three antituberculosis chemoprophylaxis regimens in patients with silicosis in Hong Kong. Hong Kong Chest Service/Tuberculosis Research Centre, Madras/British Medical Research Council. Am Rev Respir Dis. 1992; 145(1):36-41.

41. Polesky A, Farber HW, Gottlieb DJ, et al. Rifampin preventive therapy for tuberculosis in Boston's homeless. Am J Respir Crit Care Med. 1996;154(5):1473-1477.

42. Aspler A, Long R, Trajman A, et al. Impact of treatment completion, intolerance and adverse events on health system costs in a randomised trial of 4 months rifampin or 9 months isoniazid for latent TB. Thorax. 2010;65(7):582-587.

43. Menzies D, Dion MJ, Rabinovitch B, Mannix S, Brassard P, Schwartzman K. Treatment completion and costs of a randomized trial of rifampin for 4 months versus isoniazid for 9 months. Am J Respir Crit Care Med. 2004;170(4):445-449.

44. Young H, Wessolossky M, Ellis J, Kaminski M, Daly JS. A retrospective evaluation of completion rates, total cost, and adverse effects for treatment of latent tuberculosis infection in a public health clinic in central massachusetts. Clin Infect Dis. 2009;49(3):424-427.

45. Holland DP, Sanders GD, Hamilton CD, Stout JE. Strategies for treating latent multiple-drug resistant tuberculosis: a decision analysis. PLoS One. 2012;7(1):e30194.

46. Blumberg HM, Burman WJ, Chaisson RE, et al; American Thoracic Society, Centers for Disease Control and Prevention, Infectious Diseases Society. American Thoracic Society/Centers for Disease Control and Prevention/Infectious Diseases Society of America: treatment of tuberculosis. Am J Respir Crit Care Med. 2003;167(4):603-662.

47. Livengood Jr, Sigler TG, Foster LR, Bobst JG, Snider DE Jr. Isoniazidresistant tuberculosis: A community outbreak and report of a rifampin prophylaxis failure. JAMA. 1985;253(19):2847-2849.

48. Martinson NA, Barnes GL, Moulton LH, et al. New regimens to prevent tuberculosis in adults with HIV infection. $N$ Eng $J$ Med. 2011; 365(1):11-20.

49. Centers for Disease Control and Prevention (CDC). Update: Fatal and severe liver injuries associated with rifampin and pyrazinamide treatment for latent tuberculosis infection. MMWR Morb Mortal Wkly Rep. 2002;51(44):998-999.

50. cdc.gov. Managing drug interactions in the treatment of HIV-related tuberculosis Centers for Disease Control and Prevention [updated July 1, 2010]. Available from: http://www.cdc.gov/tb/publications/guidelines/ TB_HIV_Drugs/RoleofRifamycins.htm. Accessed October 2, 2012.

51. Page KR, Sifakis F, Montes de Oca R, et al. Improved adherence and less toxicity with rifampin vs isoniazid for treatment of latent tuberculosis: a retrospective study. Arch Intern Med. 2006;166(17):1863-1870.

52. Ziakas PD, Mylonakis E. 4 months of rifampin compared with 9 months of isoniazid for the management of latent tuberculosis infection: a meta-analysis and cost-effectiveness study that focuses on compliance and liver toxicity. Clin Infect Dis. 2009;49(12):1883-1889.

53. A controlled trial of daily and intermittent rifampicin plus ethambutol in the retreatment of patients with pulmonary tuberculosis: results up to 30 months. Tubercle. 1975;56(3):179-189.

54. Aquinas M, Allan WG, Horsfall PA, et al. Adverse reactions to daily and intermittent rifampicin regimens for pulmonary tuberculosis in Hong Kong. Br Med J. 1972;1(5803):765-771.

55. Poole G, Stradling P, Worlledge S. Potentially serious side effects of highdose twice-weekly rifampicin. Br Med J. 1971;3(5770):343-347.

56. Zhang Y, Mitchison D. The curious characteristics of pyrazinamide: a review. Int J Tuberc Lung Disease. 2003;7(1):6-21.

57. Gordin F, Chaisson RE, Matts JP, et al. Rifampin and pyrazinamide vs isoniazid for prevention of tuberculosis in HIV-infected persons: an international randomized trial. Terry Beirn Community Programs for Clinical Research on AIDS, the Adult AIDS Clinical Trials Group, the Pan American Health Organization, and the Centers for Disease Control and Prevention Study Group. JAMA. 2000;283(11):1445-1450.

58. Stout JE, Engemann JJ, Cheng AC, Fortenberry ER, Hamilton CD. Safety of 2 months of rifampin and pyrazinamide for treatment of latent tuberculosis. Am J Respir Crit Care Med. 2003;167(6):824-827.
59. Centers for Disease Control and Prevention (CDC). Fatal and severe hepatitis associated with rifampin and pyrazinamide for the treatment of latent tuberculosis infection - New York and Georgia, 2000. MMWR Morb Mortal Wkly Rep. 2001;50(15):289-291.

60. Jasmer RM, Saukkonen JJ, Blumberg HM, et al; Short-Course Rifampin and Pyrazinamide for Tuberculosis Infection (SCRIPT) Study Investigators. Short-course rifampin and pyrazinamide compared with isoniazid for latent tuberculosis infection: a multicenter clinical trial. Ann Intern Med. 2002;137(8):640-647.

61. Keung A, Eller MG, McKenzie KA, Weir SJ. Single and multiple dose pharmacokinetics of rifapentine in man: Part II. Int J Tuberc Lung Disease. 1999;3(5):437-444.

62. Ji B, Truffot-Pernot C, Lacroix C, et al. Effectiveness of rifampin, rifabutin, and rifapentine for preventive therapy of tuberculosis in mice. Am Rev Respir Dis. 1993;148(6 Pt 1):1541-1546.

63. Miyazaki E, Chaisson RE, Bishai WR. Analysis of rifapentine for preventive therapy in the Cornell mouse model of latent tuberculosis. Antimicrob Agents Chemother. 1999;43(9):2126-2130.

64. Nuermberger E, Tyagi S, Williams KN, Rosenthal I, Bishai WR, Grosset JH. Rifapentine, moxifloxacin, or DNA vaccine improves treatment of latent tuberculosis in a mouse model. Am J Respir Crit Care Med. 2005;172(11):1452-1456.

65. Zhang T, Zhang M, Rosenthal IM, Grosset JH, Nuermberger EL. Short-course therapy with daily rifapentine in a murine model of latent tuberculosis infection. Am J Respir Crit Care Med. 2009;180(11): 1151-1157.

66. Schechter M, Zajdenverg R, Falco G, et al. Weekly rifapentine/ isoniazid or daily rifampin/pyrazinamide for latent tuberculosis in household contacts. Am J Respir Crit Care Med. 2006;173(8): 922-926.

67. Centers for Disease Control and Prevention (CDC). Recommendations for use of an isoniazid-rifapentine regimen with direct observation to treat latent Mycobacterium tuberculosis infection. MMWR Morb Mortal Wkly Rep. 2011;60(48):1650-1653.

68. Holland DP, Sanders GD, Hamilton CD, Stout JE. Costs and cost-effectiveness of four treatment regimens for latent tuberculosis infection. Am J Respir Crit Care Med. 2009;179(11):1055-1060.

69. Horn DL, Hewlett D Jr, Alfalla C, Peterson S, Opal SM. Limited tolerance of ofloxacin and pyrazinamide prophylaxis against tuberculosis. N Engl J Med. 1994;330(17):1241.

70. Lou HX, Shullo MA, McKaveney TP. Limited tolerability of levofloxacin and pyrazinamide for multidrug-resistant tuberculosis prophylaxis in a solid organ transplant population. Pharmacotherapy. 2002;22(6):701-704

71. Ridzon R, Meador J, Maxwell R, Higgins K, Weismuller P, Onorato IM. Asymptomatic hepatitis in persons who received alternative preventive therapy with pyrazinamide and ofloxacin. Clin Infect Dis. 1997;24(6):1264-1265.

72. Younossian AB, Rochat T, Ketterer JP, Wacker J, Janssens JP. High hepatotoxicity of pyrazinamide and ethambutol for treatment of latent tuberculosis. Eur Respir J. 2005;26(3):462-464.

73. Comstock GW. Frost revisited: the modern epidemiology of tuberculosis. Am J Epidemiol. 1975;101(5):363-382.

74. Centers for Disease Control and Prevention (CDC). Trends in tuberculosis - United States, 2011. MMWR Morb Mortal Wkly Rep. 2012;61(11):181-185.

75. Centers for Disease Control and Prevention. Latent Tuberculosis Infection: A Guide for Primary Health Care Providers. Available from: http://www.cdc.gov/tb/publications/ltbi/treatment.htm. Accessed November 13, 2012.

76. Centers for Disease Control and Prevention. Latent Tuberculosis Infection: A Guide for Primary Health Care Providers. Available from: http://www.cdc.gov/tb/publications/ltbi/diagnosis.htm. Accessed November 13, 2012. 
Infection and Drug Resistance

\section{Publish your work in this journal}

Infection and Drug Resistance is an international, peer-reviewed openaccess journal that focuses on the optimal treatment of infection (bacterial, fungal and viral) and the development and institution of preventive strategies to minimize the development and spread of resistance. The journal is specifically concerned with the epidemiology of antibiotic

\section{Dovepress}

resistance and the mechanisms of resistance development and diffusion in both hospitals and the community. The manuscript management system is completely online and includes a very quick and fair peerreview system, which is all easy to use. Visit http://www.dovepress.com/ testimonials.php to read real quotes from published authors.

Submit your manuscript here: http://www.dovepress.com/infection-and-drug-resistance-journal 